Results 33 patients grew at least one new isolate of NTM: MABSC ( $\mathrm{n}=20,5$ of which grew other NTM species in temporally distinct episodes), MAC $(\mathrm{n}=12) \&$ other NTMs $(\mathrm{n}=5$, mainly M. kansasii). 51\% female. Median age at 1 st isolation 12.3 yrs (range, 4-17.3), and FEV1 79.5\% predicted (50-116\%). $10(26 \%)$ initial isolates were from BAL. For MABSC: $4(20 \%)$ had ABPA and 6 (30\%) CFRD. All patients met ATS/IDSA criteria for diagnosis and were treated in accordance with national consensus guidelines. Spontaneous clearance was seen in $100 \%$ of other NTM infections. For data on clearance and treatment of MABSC and MAC, see Table 1. 9/14 who completed NTM treatment showed culture conversion at 3 months. Only 2 children with negative cultures at 3 months went on to have subsequent positive microbiology.

Conclusion This is the first report discussing treatment success for NTM in a large paediatric cohort. Although single centre, there is a similar incidence of NTM to that reported for adult CF populations. Spontaneous clearance is more common with MAC (42\%) and other NTM infections compared to MABSC (10\%). To date $53 \%$ of treated MABSC are considered eradicated 12 months post treatment. Early culture conversion appears to be linked with treatment success. Further studies are needed to identify if a lack of early clearance should identify children appropriate for further inpatient induction therapy.

\begin{tabular}{|c|c|c|}
\hline & MABSC & MAC \\
\hline $\mathrm{n}$ & 20 & 12 \\
\hline Spontaneous clearance, $\mathrm{n}(\%)$ & $2(10 \%)$ & $5(42 \%)$ \\
\hline Commenced treatment, $\mathrm{n}(\%)$ & $17(85 \%)$ & $5(42 \%)$ \\
\hline Days to treatment from $1^{\text {st }}$ isolation, median (range) & $113(15-587)$ & $518(42-698)$ \\
\hline $\begin{array}{l}\text { Treatment duration in months, } \\
\text { mean (range) }\end{array}$ & $24.6(15-48)$ & $18.5(14-22)$ \\
\hline Completed treatment, $\mathrm{n}(\%)$ & $10(59 \%)$ & $4(80 \%)$ \\
\hline Eradication with treatment, $\mathrm{n}(\%)$ & $9(53 \%)$ & $3(60 \%)$ \\
\hline Relapsed after treatment, $\mathrm{n}(\%)$ & $2(12 \%)$ & 0 \\
\hline
\end{tabular}

\section{Innate Immunity in Lung Disease}

\section{S43 HYPOXIA UPREGULATES PI3KINASE-DEPENDENT NEUTROPHIL DEGRANULATION AND NEUTROPHIL- MEDIATED TISSUE INJURY}

${ }^{1} \mathrm{KM}$ Lodge, ${ }^{1} \mathrm{~K}$ Hoenderdos, ${ }^{1} \mathrm{AJ}$ Robbins, ${ }^{1} \mathrm{DM}$ Storisteanu, ${ }^{1} \mathrm{ER}$ Chilvers, ${ }^{1} \mathrm{~W}$ Li, ${ }^{2}$ AM Condliffe. 'University of Cambridge, Cambridge, UK; ${ }^{2}$ University of Sheffield, Sheffield, UK

\subsection{6/thoraxjnl-2016-209333.49}

Introduction Damage to host tissue from persistent neutrophilic inflammation is implicated in the pathogenesis of many diseases, including chronic obstructive pulmonary disease (COPD). Infected/inflamed tissues can be profoundly hypoxic; this state may synergise with inflammatory cytokines to promote a destructive neutrophil phenotype with enhanced potential for tissue damage.

Methods Neutrophils isolated from COPD patients or healthy volunteers were incubated under normoxia $(21 \%$ O2) or hypoxia $(0.8 \% \mathrm{O} 2)$ before treatment with priming (GM-CSF/PAF/TNF- $\alpha$ ) and stimulating (fMLP) agents, with/without PI3Kinase inhibitors (pan $/ \gamma / \delta)$. Neutrophil elastase (NE) activity was measured by Enzchek $^{\circledR}$ assay. Western blotting for total and phosphorylated Akt was performed using cell lysates. Neutrophil extracellular trap (NET) production was assessed by fluorescence absorbance. Neutrophil supernatants were incubated with primary human pulmonary artery endothelial cells (HPAEC); death and detachment were measured by MTT assay and confocal microscopy. Precipitated neutrophil supernatants were separated by SDS polyacrylamide gel electrophoresis (PAGE) and silver stained. S100A8/A9 homo- and heterodimer content of neutrophil supernatants was assessed by ELISA.

Results Hypoxia increased NE release in an agonist- and PI3K- $\boldsymbol{\gamma}$ dependent manner, with more pronounced hypoxic degranulation responses seen in exacerbating COPD patients. Hypoxia augmented resting and cytokine-stimulated Akt phosphorylation; PI3K- $\gamma$ inhibition abrogated Akt phosphorylation and prevented the hypoxic uplift of NE release. Hypoxia did not increase NET production in resting or GM-CSF/fMLP treated cells. Hypoxic neutrophil supernatants induced extensive HPAEC detachment and death, which was prevented by co-incubation with alpha-1 antitrypsin. Silver stained protein bands from precipitated neutrophil supernatants separated by SDS-PAGE were identified by mass spectrometry, suggesting a hypoxic increase in damage associated molecular pattern (DAMP) proteins S100A8 and S100A9. When interrogated by ELISA, there was no difference between the amount of S100A8/A9 hetero- or homodimers in normoxic versus hypoxic supernatants.

Conclusion Hypoxia augments neutrophil degranulation in an agonist- and PI3K- $\gamma$-dependent manner, which may be further increased during COPD exacerbations. Hypoxic neutrophil supernatants have enhanced capacity to damage endothelial cells in vitro, likely due to increased release of NE. The contribution of S100A8/A9 proteins to this damage is currently unclear. Hence, hypoxia promotes a destructive histotoxic neutrophil phenotype with potential relevance to diseases such as COPD.

\section{S44 PSEUDOMONAS AERUGINOSA INDUCES NEUTROPHIL CELL DEATH WHICH IS REVERSED BY HYPOXIA}

SP Williams, R Dickinson, SR Walmsley, MKB Whyte. University of Edinburgh, Edinburgh, UK

10.1136/thoraxjnl-2016-209333.50

Introduction Neutrophils accumulate in the lungs of patients with bronchiectasis and cystic fibrosis, and the resulting inflammation causes tissue hypoxia. Neutrophils have a unique ability to survive and function in such hypoxic environments, a response regulated by the hypoxia-inducible transcription factor (HIF)/ hydroxylase oxygen sensing pathway.

Pseudomonas aeruginosa is an opportunistic pathogen which colonises patients with chronic lung disease, including cystic fibrosis. It secretes the toxin pyocyanin, which induces neutrophil apoptosis in an oxygen-dependent manner, as a means of immune-evasion. $P$. aeruginosa has recently been shown to possess hydroxylase-homologs, suggesting that prokaryotes may also have oxygen-sensing capabilities. A prolyl-hydroxylase (PHD)deficient strain secretes higher levels of pyocyanin compared with wild-types.

We hypothesised that PHD-deficient $P$. aeruginosa induces a higher degree of neutrophil death compared with an otherwise genetically identical wild-type strain. Furthermore, we postulated that the killing effects of these bacteria are reversed in hypoxia. 
Methods Human neutrophils were purified from healthy volunteers by discontinuous percoll gradients. PAO-1 and PHD-deficient strains were grown in lysogeny broth and equivalent growth curves confirmed. Supernatants from wild-type and PHD-deficient $P$. aeruginosa were harvested at two hours and then co-cultured with neutrophils in normoxia and hypoxia. Neutrophil viability and apoptosis was then assessed using AnnexinV/To-pro3 staining on flow cytometry at three and five hours.

Results Control neutrophils in normoxia saw a decrease in viability of $6 \%$ between three and five hours. Neutrophils treated with supernatant from the PHD-deficient strain experienced a decrease in viability from 3139 ( \pm 968 ) cells at three hours to $2058( \pm 586)$ at five hours - a decline of 34\% ( $\mathrm{P}<0.05)$. Normoxic neutrophils treated with the wild-type strain, however, saw a decrease of $21 \%(\mathrm{P}<0.05)$.

Hypoxic conditions reversed the killing effects of wild-type $P$. aeruginosa: after five hours neutrophils in normoxia experienced a $21 \%$ decrease in viability, whereas the viability of hypoxic cells only decreased by $8 \%(\mathrm{P}<0.05)$.

Discussion These data highlight the relationship between tissue oxygen tensions and host immunity and that bacteria have evolved virulence factors with novel mechanisms of action; namely preventing neutrophil survival at sites of inflammation. Moreover, the potential oxygen-sensing capabilities of prokaryotes are intricately linked to bacterial virulence.

\section{S45 EVALUATING THE SENSITIVITY AND SPECIFICITY OF ACTIVE NEUTROPHIL ELASTASE AS A BIOMARKER FOR BACTERIAL INFECTION IN SUBJECTS WITH COPD}

\author{
${ }^{1} \mathrm{~S} J$ Thulborn, ${ }^{2} \mathrm{~N}$ Akram, ${ }^{3} \mathrm{~V}$ Mistry, ${ }^{3} \mathrm{CE}$ Brightling, ${ }^{4} \mathrm{~K}$ Moffitt, ${ }^{4} \mathrm{D}$ Ribeiro, ${ }^{1} \mathrm{M}$ Bafadhel. \\ ${ }^{1}$ University of Oxford, Oxford, UK; ${ }^{2}$ Oxford Brookes University, Oxford, UK; ${ }^{3}$ University of \\ Leicester, Leicester, UK; ${ }^{4}$ ProAxsis Ltd, UK, Carterton
}

\subsection{6/thoraxjnl-2016-209333.51}

Introduction COPD is a neutrophilic disease, with the majority of subjects having a sputum neutrophil percentage of $>60 \%$. Neutrophil elastase (NE) is a serine proteinase, secreted by neutrophils and macrophages during inflammation and has a role in the destruction of bacteria within the host. New advancements now allow accurate assessment of active protease levels in complex biological samples. We sought to investigate if active NE could be used as a biomarker for bacterial infection in subjects with COPD.

Methods NE was quantified using ProteaseTag ${ }^{\text {TM }}$ active NE Immunoassay (ProAxsis, Belfast) from cell-free sputum supernatant from 31 COPD subjects (20 Males; mean age 65, range 45 to 81$)$ at stable state and during an exacerbation. Bacterial infection was defined as $\geq 10^{7} \mathrm{CFU} / \mathrm{mL}$ in sputum. Subject demographics, sputum cell differential counts and polymerase chain reaction (PCR) for respiratory pathogens were measured.

Results Active NE was higher during an exacerbation compared to stable state (fold difference (95\% CI) 0.50 (0.22 to 0.78), $\mathrm{p}=0.001$ ) (Figure 1). NE correlated with total sputum neutrophils ( $\mathrm{p}<0.0001, \mathrm{r}=0.48$ ) and total bacterial load measured by $\mathrm{CFU} / \mathrm{mL}(\mathrm{p}<0.01, \mathrm{r}=0.39)$ and $\mathrm{qPCR}(\mathrm{p}<0.05, \mathrm{r}=0.33)$. When looking at the main respiratory pathogens no correlations were seen between $H$. influenzae ( $\mathrm{p}=0.43, \mathrm{r}=-0.11$ ), S. aureus $(\mathrm{p}=0.34, \mathrm{r}=-0.14)$ or $\mathrm{S}$. pneumonia $(\mathrm{p}=0.11$, $\mathrm{r}=0.23$ ); however a correlation was seen between NE and $M$. catarrhalis ( $\mathrm{p}=0.01, \mathrm{r}=0.36$ ). NE has an area under the receiver operator curve of 0.72 [0.58 to 0.85$]$ to identify a bacterial infection with a sensitivity and specificity of $67.74 \%$ and $67.86 \%$ at a NE cut off of $2335 \mathrm{ng} / \mathrm{mL}$.

Conclusion Active NE is elevated during a COPD exacerbation compared to baseline. Active NE is associated with neutrophilic inflammation and bacteria; and may be a viable biomarker for bacterial infection in COPD.

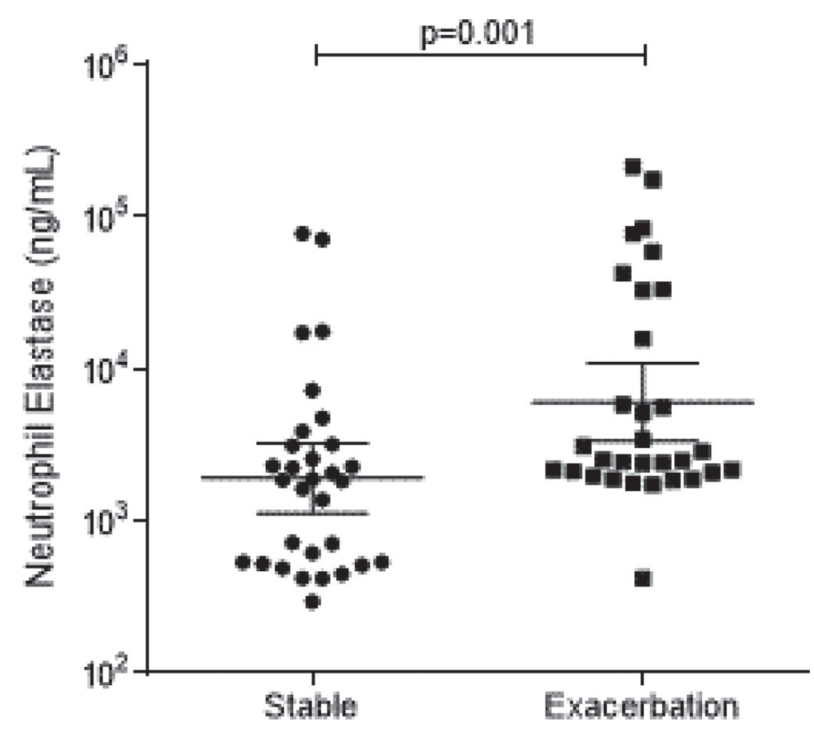

Abstract S45 Figure 1 Sputum active NE levels at stable and exacerbation state from 31 paired COPD subjects. Mean and $95 \% \mathrm{Cl}$

\section{S46 NEUTROPHIL VASCULAR ENDOTHELIAL GROWTH FACTOR (VEGF) AS A DRIVING FORCE FOR ANGIOGENESIS IN BRONCHIECTASIS?}

${ }^{1} \mathrm{CC}$ Cole, ${ }^{2} \mathrm{SC}$ Carnell, ${ }^{3} \mathrm{KJ}$ Jiwa, ${ }^{4} \mathrm{JB}$ Birch, ${ }^{1} \mathrm{KH}$ Hester, ${ }^{1} \mathrm{CW}$ Ward, ${ }^{1} \mathrm{JS}$ Simpson, ${ }^{2} \mathrm{ADS}$ De Soyza. Institute of Cellular Medicine, Newcastle University, Newcastle Upon Tyne, UK; ${ }^{2}$ Newcastle Clinical Trials Unit, Newcastle Upon Tyne, UK; ${ }^{3}$ Sir William Leech Centre for Lung Research, Freeman Hospital, Newcastle Upon Tyne, UK; ${ }^{4}$ Institute for Cell and Molecular Biosciences, Newcastle Upon Tyne, UK

\subsection{6/thoraxjnl-2016-209333.52}

Introduction Bronchiectasis (BR) in a pulmonary disease thought to involve a characteristic dilation of the bronchi resulting from a cycle of airway infection and inflammation. This inflammation is believed to be driven by neutrophils, which are present in the BR lung in high number. Vascular endothelial growth factor (VEGF) is a pro-angiogenic cytokine that may be upregulated in BR and could contribute towards creating a pro-angiogenic airway environment by supporting neutrophil migration into the airway tissue, however this has yet to be shown.

Aims 1) Examine the BR airway for any indications of increased angiogenesis, 2) Assess the ability of neutrophils to secrete VEGF upon stimulation in vitro, 3) Evaluate sera/sputa samples VEGF concentration to determine if VEGF could act as a biomarker for BR severity.

Methods Healthy volunteer (HV) and BR endobronchial biopsies were stained with a HRP conjugated anti-CD31 antibody, allowing blood vessels to be counted in a blinded manner. Peripheral blood neutrophils isolated from $\mathrm{HV}$ were stimulated (e.g. with TNF- $\alpha$ or bacterial PAMPs) for 4 hours, VEGF levels in supernatants were then quantified using ELISA. A VEGF ELISA was also used to determine VEGF concentration in sera and sputa samples 